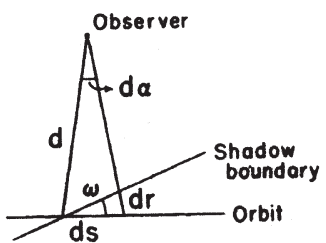
Fig. 2. The error in height as computed from the error in position.
$\mathrm{d} s \approx(2 \pi \mathrm{d} / 360) \mathrm{d} \alpha ; \mathrm{dr} \approx \tan \omega \mathrm{ds}$. With $\omega \max .=27 \cdot 3^{\circ}$ and $\mathrm{d}=800 \mathrm{~km} ., \mathrm{d} r / \mathrm{d} \alpha=7.2 \mathrm{~km} . / \mathrm{deg}$

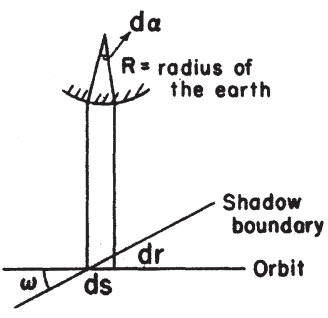

Fig. 3. The error in height as computed from the error in time. In the time dt (in min.) a point on the Earth's surface at latitude $\varphi$ has moved by the amount ds $=2 \pi / 360 R \cos \varphi \mathrm{d} \alpha=2 \pi / 360$ $R \cos \varphi \mathrm{d} t / 4 . \mathrm{d} r=\tan \omega \mathrm{d} s$. $\mathrm{d} r / \mathrm{d} t=10 \cdot 8$

Observations of a shadow-egress give very accurate values for the height of a satellite above the Earth's surface. At a height of $800 \mathrm{~km}$. the largest angle possible between the orbit and the boundary of the

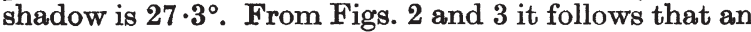
error in the observed position of egress of one degree results in a maximum error of $7.2 \mathrm{~km}$. in the distance. An error of one minute in time results in an error of $10.8 \mathrm{~km}$. in distance. Therefore, even approximate observations give rather accurate values for the height of a satellite, at least within $\pm 30 \mathrm{~km}$. The height of $1,000 \mathrm{~km}$. which was expected would require that the satellite had already left the shadow

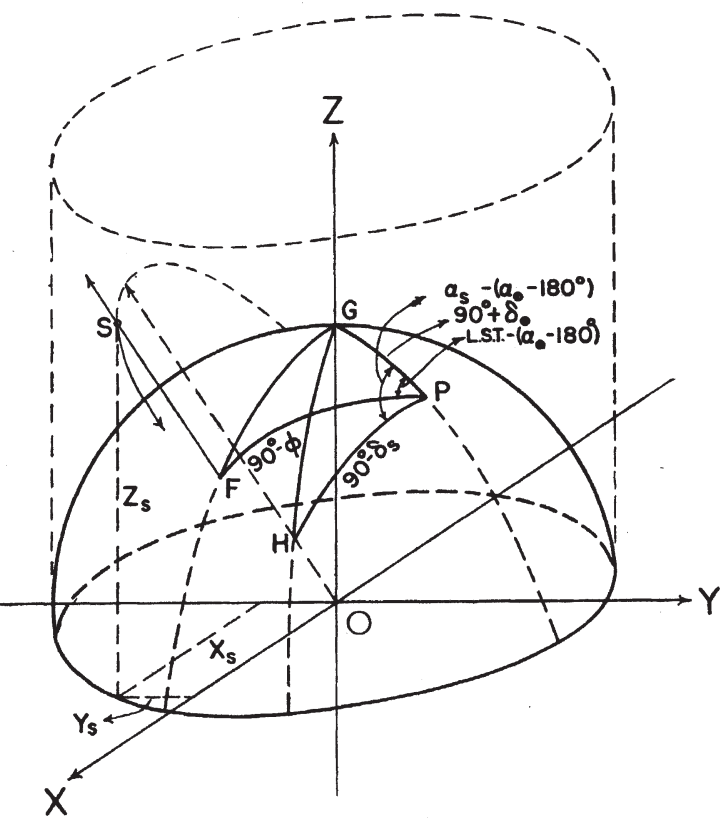
Fig. 4. Derivation of the height of a satellite above the Earth's
surface. $F$, observer; $P$, pole of the Farth; $G$, point opposite surface. $F$, observer; $P$, pole of the Earth; $G$, point opposite to the Sun; $H$, point with the observed egress position in the zenith ; $S$, satellite leaving the shadow ; $\phi_{s}$ observer's latitude ;
of $\delta ;$, right ascension and declination of the observed position
of $S ; \alpha \odot, \delta \odot$, right ascension and declination of the Sun; L.S.T. local sidereal time at a distance of $30^{\circ}$ from our first observed position. There seems to be no reason why the satellite should not have been visible if this were correct.

The method used for the derivation is shown in Fig. 4. From the observed position of egress, the sidereal time, the latitude of the observer, and the spherical coordinates of the Sun, rectangular co-ordinates can be derived for the points $F$ and $H$. The equations for the line $\overline{O H}$ give the direction toward the observed position of egress. The intersection of a line through $F$ parallel to $\overline{O H}$ with the cylindrical or conical shadow gives the rectangular co-ordinates $X_{8}, Y_{8}, Z_{8}$ for the satellite leaving the shadow. The distance from the Earth's centre is then $r=\left(X_{8}^{2}+Y_{8}^{2}+Z_{8}^{2}\right)^{1 / 2}$. HeINZ NeCKEL

Warner and Swasey Observatory, East Cleveland 12,

Ohio. Dec. 21.

\section{Precision Determination of the Velocity of Electromagnetic Waves}

The free-space phase velocity of electromagnetic waves in vacuo has been measured by means of a millimetre-wave interferometer operating at a frequency of $72 \mathrm{Gc} / \mathrm{s}$., corresponding to a wave-length of about $4 \mathrm{~mm}$. The result is :

$$
c_{0}=299792 \cdot 50 \pm 0.10 \mathrm{~km} . / \mathrm{s} \text {. }
$$

The variation represents the standard deviation of a single observation in statistical combination with estimated systematic uncertainties, the greatest of these being in the measurement of the standards of length used (derived by optical interferometry), and in the measurement of the refractive index of the air surrounding the microwave interferometer (obtained by means of a microwave refractometer).

The 24-Gc./s. prototype instrument has already been described ${ }^{1}$, the 72 -Gc./s. interferometer being basically identical, but considerably improved in performance. A full report of the present determination will be published in due course, but it should be noted here that the value previously assigned to the one-metre length-standard used with the prototype interferometer was too great, and this has caused the published value of $c_{0}$ obtained from that instrument to be high by $0.2 \mathrm{~km}$./s. Thus the corrected value of the earlier determination ${ }^{1}$ is : $c_{0}=299792 \cdot 7_{\mathrm{s}} \pm 0.3 \mathrm{~km} . / \mathrm{s}$.

This work has been carried out as part of the research programme of the National Physical Laboratory and is published by permission of the Director of the Laboratory.

Metrology Division,

K. D. Froome

National Physical Laboratory,

Teddington, Middlesex. Nov. 5.

${ }^{1}$ Froome, K. D., Proc. Roy. Soc., A, 223, 195 (1954).

\section{Metallographic Studies of Iron Carbide and Tungsten Carbide}

IN most metallographic investigations of steels or of cemented carbides the fine details of the carbides are not studied. We have just completed an etching study of iron carbides made in three different ways and of some tungsten carbide (1/32-in. equilateral triangles) single crystals. The iron carbide studies, made with Carapella's reagent (5 gm. ferric chloride, 